Tropical Journal of Pharmaceutical Research January 2015; 14 (1): 79-85

ISSN: $1596-5996$ (print); 1596-9827 (electronic)

(C) Pharmacotherapy Group, Faculty of Pharmacy, University of Benin, Benin City, 300001 Nigeria.

All rights reserved.

Available online at http://www.tjpr.org

Original Research Article

http://dx.doi.org/10.4314/tjpr.v14i1.12

\title{
Systemic Effect of Angipars on Regulation of Wound Healing is Mediated by CXC in Diabetes
}

\author{
Farangis Fatehi ${ }^{1}$, Gholamhossein Hassanshahi ${ }^{2}$, Seyed Ebrahim Hosseini ${ }^{3}$, \\ Ahmad Shabani Zade ${ }^{4}$ and Mohammad Mohsen Taghavi ${ }^{4 *}$ \\ ${ }^{1}$ Physiology-Pharmacology Research Center, ${ }^{2}$ Molecular Medicine Research Center, ${ }^{3}$ Department of Biology, Fars Sciences \\ and Research Branch, Islamic Azad University, Fars, ${ }^{4}$ Department of Anatomy, School of Medicine, Rafsanjan University of \\ Medical Sciences, Rafsanjan, Iran
}

*For correspondence: Email: taghavi164@yahoo.com; Tel: +98-391-4220244, +98-913-391-3618; Fax: +98-391-5225209

Received: 19 January 2014

Revised accepted: 16 November 2014

\begin{abstract}
Purpose: To measure CXCL10 as angiostatic, and CXCL1, CXCL12 as angiogenic chemokines in the tissues of wounds of diabetics following treatment with insulin, angipars (a herbal Iranian drug) and a combination of angipars and insulin.

Methods: Forty eight male Wistar rats weighing 200 - $250 \mathrm{~g}$ were used. The induction of diabetes was carried out with $50 \mathrm{mg} / \mathrm{kg}$ of STZ (streptozotocin). Approximately, 56 days following the induction of diabetes, the rats were injured to establish wound lesion. They were divided into four main groups: nondiabetic control group (received only saline), diabetes group without treatment (received only saline), diabetes group which received insulin (reference) as treatment, and diabetes group which received both insulin and angipars. After 12 days of treatment, the animals were subjected to blood sampling from retro-orbital vein and CXC chemokines were analyzed by Western blotting.

Results: The results show that the concentration of CXC10 decreased from $95 \mathrm{pg} / \mathrm{ml}$ in the diabetic control group to 40 and $10 \mathrm{pg} / \mathrm{ml}$ in the insulin and combined angipars/insulin groups, respectively ( $p \leq$ 0.05). However, CXCL12 concentration was not changed among the various groups compared to the control group. In diabetic control and angipars-insulin groups, CXCL1 level (pg/ml) was 98 and 50, respectively, thus indicating that expression of CXCL1 chemokine decreased significantly $(p \leq 0.05)$. Conclusions: Angipars, due probably to its richness in some natural compounds such as coumarin and flavonoids (which are antioxidants), mediates chemokines expression and may be effective in the regulation of angiogenesis and inflammation via balancing of chemokines expression.
\end{abstract}

Keywords: Diabetes mellitus, Angipars, Insulin, Chemokine, Angiostatic, Angiogenic

Tropical Journal of Pharmaceutical Research is indexed by Science Citation Index (SciSearch), Scopus, International Pharmaceutical Abstract, Chemical Abstracts, Embase, Index Copernicus, EBSCO, African Index Medicus, JournalSeek, Journal Citation Reports/Science Edition, Directory of Open Access Journals (DOAJ), African Journal Online, Bioline International, Open-J-Gate and Pharmacy Abstracts

\section{INTRODUCTION}

There is abundant evidence that both environmental and genetic parameters influence diabetes type 2 (T2D) [1]. Diabetes is also considered an immune-mediated disease with changed cytokines expression pattern. For example, in T2D, peripheral blood monocytes are increased which confirms the involvement of an inflammatory reaction in its pathogenesis [2]. Activation of inflammatory pathways and oxidative stress induce devastating effects on the tissues in diabetes mellitus (DM). As an instance, impaired vision and functions of organs such as liver and kidney, cardiovascular dysfunction, neuropathy, impaired processes of healing of wounds and impaired stomach and gut functions are frequent in DM [3]. The phenomenon of 
wound healing in diabetic foot syndrome depends on the ability to produce a series of growth factors and consequently launch appropriate response to them [4]. Angipars (AP) is an Iranian herbal drug that has been considered for treatment of diabetes due to the fact that it contains two major groups of phenolic compounds including 7 - hydroxy-coumarin and flavonoids. These compounds have antioxidant properties [5].

Cytokines and chemokines are key mediators in the regulation of angiogenesis and angiostatis [6]. Chemokines are a subgroup of cytokines with several biological effects such as angiogenesis, and angiostatic properties. Stromal derived factor 1 (SDF-1), also known as CXCL12, fits into the CXC group. The key roles of CXCL12 as an inhibitory chemokine in autoimmunity and inflammation raise questions concerning the impacts of this chemokine in the pathogenesis of some diseases including T2D [7]. CXCL10 or IP. 10 is expressed by infected cells during insult and injury as the main component of proinflammatory immune responses [6]. The growthrelated oncogene alpha (GRO- $\alpha$ ) or CXCL1, is one of the main chemokines in angiogenesis and pro-inflammatory immune responses. CXCL1 is mainly involved in chemo-attraction of neutrophil into the insulted tissues [6].

Thus, considering the point that in DM complications most organs are involved and functionally impaired, we hypothesized that AP having angiogenesis properties might be effective in the treatment of diabetic foot syndrome (DFS) complications. Therefore, this study was aimed to explore the regulatory effects of AP on DFS via expression of some CXC chemokines including CXCL1, CXCL10, and CXCL12 in streptozocin-induced DM in the rat model of DFS.

\section{EXPERIMENTAL}

\section{Animals}

In this study, 48 male Wistar rats (aged 8 weeks and weighing $250-300 \mathrm{~g}$ ) were purchased from Pasteur Institute-Iran. Animals had free access to food and water with standard conditions and were housed at $23 \pm 2{ }^{\circ} \mathrm{C}$ and $12 \mathrm{~h}$ light /dark cycle. Procedures involving animals and their care were conducted in accordance with the Guide to the Care and Use of Experimental Animals [8]. Approval from the institutional animal ethics committee was also obtained approval (ref no. 1159).

\section{Induction of DM}

The induction of DM was carried out by intramuscular injection of $50 \mathrm{mg} / \mathrm{kg}$ of STZ (streptozotocin) in a region between the two ears of the animal. Subsequent to the induction of diabetes, symptoms of diabetes such as overeating, overdrinking, urination and weight loss were observed. The level of blood glucose was measured after 5 days of DM induction using a glucometer device and animals having a blood glucose level above $300 \mathrm{mg} / \mathrm{dl}$ were considered as diabetic and subjected to further examinations. All rats were kept for 8 weeks until chronic diabetes symptoms were obviously observed and then subjected to further analysis.

\section{Treatments}

Approximately 56 days following the induction of DM, rats were divided into four main groups (with 12 rats in each group) as following: Group I: non-diabetic control group (received only saline), Group II: diabetic group without treatment (received only saline), Group III: diabetic group treated only with insulin, group IV: diabetic group treated with both $\mathrm{AP}$ and insulin. Treatment (either normal saline or drugs) were injected to the animals at specified doses $(0.1 \mathrm{mg} / \mathrm{kg})$. Angipars was purchased from ParsRoos Co. (Tehran, Iran). AP was freshly diluted in sterile saline (1:10) and was injected intra-peritonealy to Group IV. In groups III and IV, Protamine insulin was injected (4-6 U/Kg/Day Sc). After the last injection, animals were anesthetized, and specimens were collected and used for further examinations.

\section{Analysis of chemokines by Western blotting}

Proteins were extracted from freeze-dried, dissected and homogenized diabetic wound samples using lysis buffer (Cell Signaling Technology, Beverly, MA, USA).

Samples were used for sodium dodecyl sulfatepolyacrylamide gel electrophoresis (SDS PAGE). Immunoblotting and densitometry were performed to quantify the expressions of CXCL1, CXCL10 and CXCL12. Equal amounts of protein (35 mg) were loaded and resolved on a $10 \%$ SDS-PAGE and then transferred to a nitrocellulose membrane. After blocking with $3 \%$ (w/v) milk in Tween (10 mM Tris, pH 7.4 containing $140 \mathrm{mM} \mathrm{NaCl}, 0.1 \%$ [v/v] Tween 20), the nitrocellulose membrane was incubated overnight at $4{ }^{\circ} \mathrm{C}$ in PBS/Tween containing $3 \%$ w/v milk including anti-rat CXCL1, CXCL10, CXCL12 (R\&D system, UK) and $\beta$-actin monoclonal antibody (Sigma, Mo, USA) 
Subsequently, anti-mouse horseradish peroxidase-conjugated antibodies(Amersham Life Science, UK) (diluted, 1:1000) were used, and accordingly the enhanced chemiluminescence (ECL) detection system (Amersham Life Science, UK) was used to define protein localization and amount. $\beta$-actin was used as an internal control to compare the data from different films.

\section{Densitometry}

The densitometry of bands on X-ray film was determined by densitometry analysis on Model GS-700 Imaging Densitometer using Molecular Analyst ${ }^{\circledR}$ software. The range of linearity of detection by autoradiography was assessed before any results were analyzed.

\section{Isolation of peripheral blood leukocytes}

Venous blood, from healthy adult volunteers, was aseptically collected in sterile tubes containing preservative-free sodium heparin (25 units/ml of blood). Heparinised blood was kept at room temperature before processing. Peripheral white blood cells were isolated within two hours of blood collection to ensure a good viability and high cell recovery. Peripheral white blood cells were isolated by a modification of the method of Boyum [9]. Heparinized blood was diluted (1:1) in washing medium [RPMI 1640 supplemented with $10 \%$ (v/v) FCS (fetal calf serum)]. Diluted blood samples were layered on top of lymphoprep (Ficoll-Hypaque of specific gravity 1077) in a large falcon $(50 \mathrm{ml})$ tube and this was centrifuged at $950 \mathrm{~g}$ for $20 \mathrm{~min}$ at room temperature (Beckman, Allegra ${ }^{\mathrm{TM}} 21$ centrifuge, USA).The clear tiny white layer interface between milky plasma and Ficoll contains peripheral blood leukocytes. These cells were collected either by removing the supernatant first or directly from the interface by piercing a pipette through the clear supernatant with minimum contamination of Ficoll. Cells were then transferred into a new sterile tube and re-suspended in a small amount of washing medium, and the tube was filled with the washing medium and centrifuged at $400 \mathrm{~g}$ for a further $10 \mathrm{~min}$ at room temperature. The supernatant was discarded and the pellet was gently re-suspended in washing medium for a second wash. Cells in the pellet were harvested by centrifugation at $400 \mathrm{~g}$ for $10 \mathrm{~min}$. The supernatant was discarded and the pellet was gently re-suspended in final wash medium, consisting of RPMI 1640 supplemented with 10 $\% \quad(\mathrm{v} / \mathrm{v})$ FCS and antibiotics (streptomycinpenicillin 10,000 IU/ml, Sigma UK) and was recentrifuged at $400 \mathrm{~g}$ for $10 \mathrm{~min}$. After the final wash, the supernatant was discarded and the pellet was re-suspended in the desired amount (2 - $5 \mathrm{ml}$, depending on pellet size) of complete medium consisting of RPMI 1640 supplemented with $10 \%(\mathrm{v} / \mathrm{v})$ of FCS fetal calf serum, $2 \mathrm{mM}$ Lglutamine and antibiotics (penicillin-streptomycin $10,000 \mathrm{IU} / \mathrm{ml})$. Total cell count was then performed (Casy I cell counter + analyzer system model SCÄREF System $\mathrm{GmbH}$ ). The total cells were adjusted to $0.5 \times 10^{6} / \mathrm{ml}$ and chemotaxis assay was performed as in the following section.

\section{In vitro chemotaxis assay}

Migration of peripheral blood leukocytes was assessed using a 48 well micro-chemotaxis technique. The two compartments were separated with a polycarbonate (polyvinylpyrrolidone-free) filter (6.5 $\mu \mathrm{m}$ pore size, Corning Incorporation). Transwell chambers were positioned face up, with the membranes pre-coated with fibronectin $(10 \mu \mathrm{g} / \mathrm{ml}$ for $1-3$ hour in an atmosphere of air: $5 \% \mathrm{CO}_{2}$ and $37^{\circ} \mathrm{C}$ ). After incubation with fibronectin, the fibronectin solution was removed and the chamber membrane was blocked (for 30-45 min) with heat-inactivated BSA $(10 \mathrm{mg} / \mathrm{ml}$ BSA in PBS, after treatment at $85^{\circ} \mathrm{C}$ for $\left.13 \mathrm{~min}\right)$. Membranes were subsequently washed twice with PBS to eliminate residual BSA. Medium supernatant $(500 \mu \mathrm{l})$ obtained from hepatocyte culture was placed into the lower compartment of each Transwell. The upper compartment was filled with $500 \mu \mathrm{l}$ of human peripheral white blood cells suspended in their appropriate culture medium. Cultures were continued at $37{ }^{\circ} \mathrm{C}$ in an atmosphere of air: $\mathrm{CO}_{2}$ for various periods. At this stage, two different fractions of cells were assessed, one fraction being the cells that were attached to the polycarbonate membrane and the second fraction was the cells that passed through the membrane into the lower chamber (passed cells). At the end of incubation, membranes, with attached cells, were washed 3 times with PBS. Membrane-attached cells were fixed by addition of methanol to the membranes (for 2 - $5 \mathrm{~min}$ ) and then washed 3 times with PBS. Fixed membrane-attached cells were stained either with crystal violet $(0.1 \% \mathrm{w} / \mathrm{v}$ in double distilled water) for 15 - $20 \mathrm{~min}$ or with Jenner-Giemsa's. For staining of the fixed membranes using Jenner-Giemsa's method, membrane were stained first in Jenner solution (a solution of $0.3 \% \mathrm{w} / \mathrm{v}$ Jenner stain in $70 \%$ methanol) for $2 \mathrm{~min}$ and then by addition of an equal portion of PBS ( $\mathrm{pH} \mathrm{6.4)} \mathrm{for} \mathrm{a} \mathrm{further} 1 \mathrm{~min}$. The Jenner solution was removed and Giemsa's stain (Giemsa's stain $2 \% \mathrm{v} / \mathrm{v}$ in $\mathrm{pH}$ 6.4 PBS) was added for $30 \mathrm{~min}$ and at the end of incubation membranes were washed by three changes in PBS (pH 6.4) and air-dried. Membranes were cut 
and mounted for further analysis. Membranes were mounted as following: one drop of Loetite 357 resin was placed onto the membrane, and then a coverslip was applied and firmly pressed down. A permanent mount was achieved by exposing the membrane to ultraviolet light from a lamp held in close proximity to the slide for about $30 \mathrm{~s}$. Membrane-attached cells were counted in 3 high power fields (400x) under light microscopy, after coding the samples. Membrane-permeant cells were counted by light microscopy and/or by size gating with an automated counter system (Casy I cell counter + analyzer system model SCÄREF System $\mathrm{GmbH}$ ).

\section{Statistical analysis}

Data were analyzed using SPSS (Version 17) (SPSS Inc., Chicago, IL, USA). Comparing variables was performed using unpaired Student's t- test. Some results were expressed as mean \pm SEM (standard error of the mean). Further, statistical differences between Western blot results were measured by Mann- Whitney U-test, where appropriate; $p<0.05$ was considered to be statistically significant.

\section{RESULTS}

Current results demonstrated that the CXCL1 level was decreased in AP and insulin treated groups and the concentration of this chemokine declined compared to control and regulatory effect was exceeded by AP (Fig 1). In these experiments the expression of chemokines at control level was assigned as $100 \%$ and other groups were related to that. The average concentration of CXCL1 was $98 \pm 2,80 \pm 6$, and $50 \pm 3 \%$ in diabetic control group, insulin group, insulin and AP group, respectively (Fig 1). The most significant difference was observed between diabetic control group and the group treated with combination of AP and insulin, which suggests the regulatory effect of $A P$ on this chemokine.

The results also demonstrated that the concentration of CXCL10 was decreased in the treatment groups and was significantly diminished in the group treated with $A P$ and insulin (Fig 2). The mean concentration of CXCL10 was $95 \pm 4 \%, 40 \pm 8 \%$ and $10 \pm 5 \%$ in the diabetic control group, group treated with insulin and the combined treatment group AP and insulin, respectively (Fig 2 ). The most significant difference was between the diabetic control group and diabetic group treated with combination of the AP and insulin.
We did not observe altered level of CXCL12 expression and its concentrations did not alter much in the different groups in comparison to control. The mean concentrations of CXCL12 was $97 \pm 1 \%, 95 \pm 4 \%$, and $98 \pm 1 \%$ in diabetic control group, insulin treated and the combined treatment group (AP and insulin), respectively. These findings in a way revealed that AP had no regulatory effect on CXCL12 expression [Fig 3].
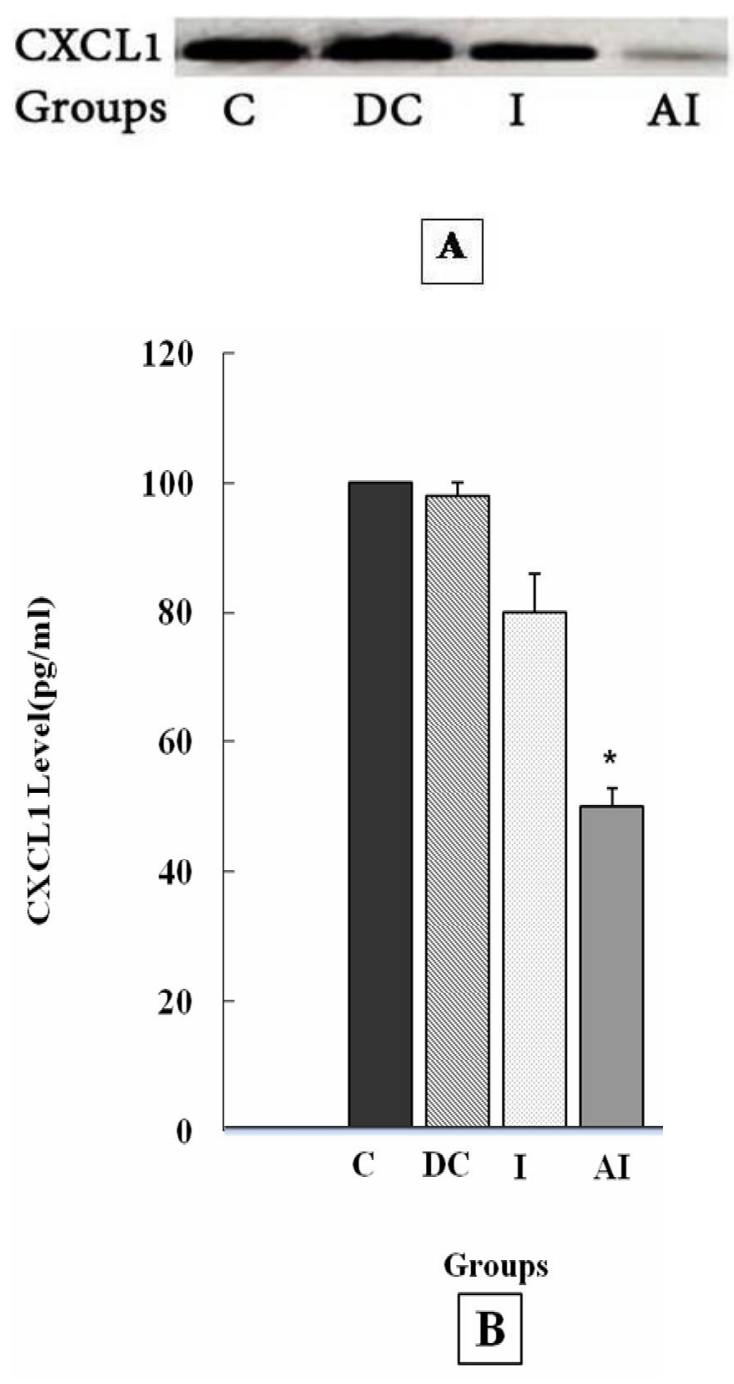

Fig 1: A) upper panel: representation profile of protein bands from Western blotting. Lane $C(1)=$ nondiabetic control, lane DC (2) = diabetic control, lane I (3) = insulin treated, lane $\mathrm{Al}(4)=$ angipars-insulin treated; B) lower panel; the expression CXCL1 in nondiabetic control (group C) was assigned as $100 \%$ of expression and expression of CXCL1 in other treatment groups was related to $100 \%$ value; *significant difference $(p<0.05)$ between C, DC and I group 


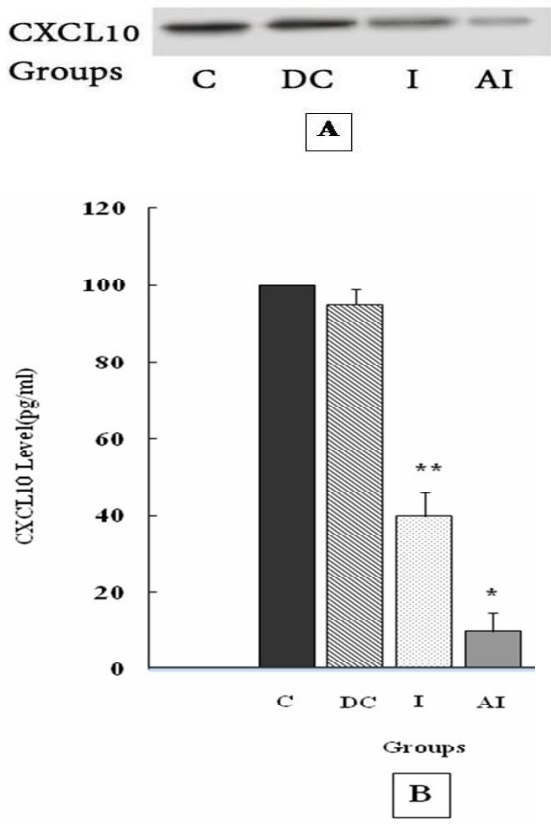

Fig 2: A) upper panel: representation profile of protein bands from Western blotting. Lane $C(1)=$ nondiabetic control, lane DC (2) = diabetic control, lane I (3) = insulin treated, lane $\mathrm{Al}(4)=$ angipars-insulin treated; B) lower panel: the expression CXCL10 in non-diabetic control (group c) was assigned as $100 \%$ of expression and expression of CXCL10 in other treatment groups was related to $100 \%$ value. *significant difference $(p<0.05)$ between $\mathrm{DC}$ and $\mathrm{Al}$ groups; ${ }^{* *}$ significant difference $(p<0.05)$ between DC and I groups

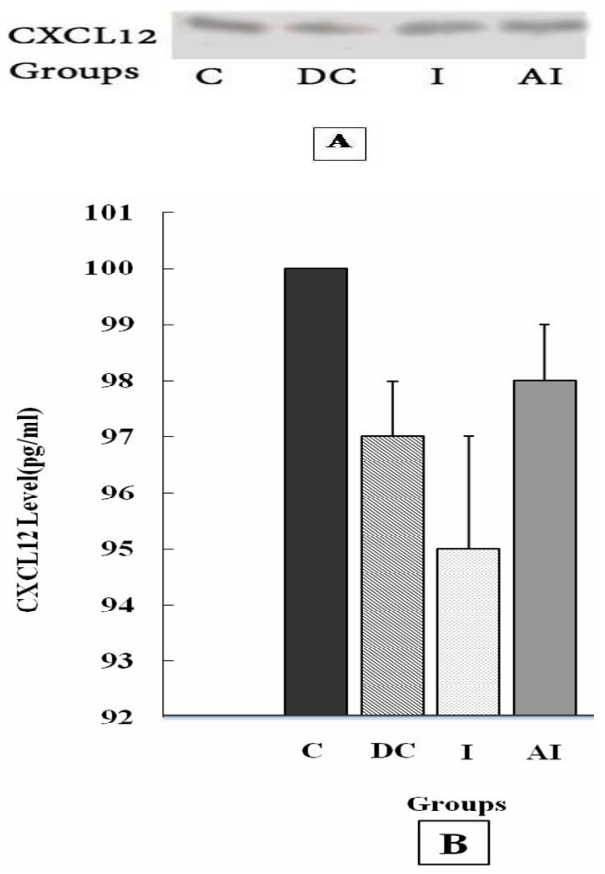

Fig 3: A) upper panel: representation profile of protein bands from Western blotting. Lane C (1) = nondiabetic control, lane DC (2) = diabetic control, lane I
(3) = insulin treated, lane $\mathrm{Al}(4)=$ angipars-insulin treated; B) lower panel: the expression CXCL12 in non-diabetic control (group C) was assigned as $100 \%$ of expression and expression of CXCL12 in other treatment groups was related to $100 \%$ value

Analysis of profile of recruited cells to the ulcers of diabetic rats demonstrated that the number of neutrophils in the ulcer lesion was $3821 \pm 183$ cell $/ \mathrm{ml}, 3503 \pm 379 \mathrm{cells} / \mathrm{ml}, 2532 \pm 385 \mathrm{cells} / \mathrm{ml}$ and $2429 \pm 290$ cells $/ \mathrm{ml}$ in non-diabetic control, diabetic control, insulin and angipars-insulin groups, respectively. The numbers of neutrophils in both of treatment groups were significantly increased in comparison to control (data not shown). Current results also indicated that the number of lymphocytes in ulcerative lesions was $587 \pm 47 \mathrm{cells} / \mathrm{ml}, 570 \pm 37 \mathrm{cells} / \mathrm{ml}, 1479 \pm 43$ cells $/ \mathrm{ml}$ and $3633 \pm 151 \mathrm{cells} / \mathrm{ml}$, in non-diabetic control, diabetic control, and insulin and angipars-insulin groups, respectively. Similarly as in neutrophils, the number of lymphocytes in both treated groups significantly decreased compared to diabetic control (587 \pm 47 cells $/ \mathrm{ml}$ and $570 \pm$ 37 cells/ml).

\section{DISCUSSION}

Doxidative stress is a predominantly important parameter involved in the pathogenesis of DM. Evidences are in favor of the fact that defective (either enzymatic or non-enzymatic) scavenging of free radicals in DM patients increases lipid peroxidation and causes multiple and invasive organ injuries. With regard to this, one of these complications is FDS in which recurrence and resistance to healing occur in FDS wounds. In these patients processes of wound healing take months and even years. In fact patients are at risk of various infections including osteomyellitis [10]. AP is a newly developed herbal compound which was recently introduced by the Iranian ministry of health as a therapy for DM complications and consists of two main groups of phenolic materials including, 7-hydroxy coumarin and flavonoids. Having these compounds, its composition made AP as a compound with antioxidative properties. Flavonoids include isorhamnetin, kaempferol and quercetin. Glycosides have beneficial properties especially for the treatment of DM patients. For instance, isorhamnetin inhibits aldosereductase and kaempferol has hypoglycemic properties and shown to increase absorption of glucose by muscle cells in healthy rats. Quercetin has been shown to decrease glucose in one hand, increase plasma insulin in other hand in STZ induced diabetic rats [11]. The present study was undertaken to verify whether CXC chemokines 
expression is regulated by AP and insulin in rat model of STZ-induced DM using western blotting analysis.

Results of our study exhibited that CXCL10 was more expressed in control group, while CXCL10 was down-regulated, when STZ induced diabetic rats were treated with the combination of insulinangipars (AI) (Fig 2). CXCL10 is an inflammatory chemokine, and increases in DM (due to inflammations raised by oxidative stresses in DM). Based on previous reports that were well supported that inflammations are mediated by IFN-y, it could be speculated that AP is probably able to control CXCL10 expression via its relative antioxidant properties. CXCL10 is a widely studied chemokine. Hassanshahi et al [10] demonstrated its in vitro induction in the presence of pro-inflammatory cytokines, including IFN-y and TNF- $\alpha$ in cultured rat hepatocytes [12]. Additionally, they also indicated that in the presence of pro-inflammatory cytokines (such as TNF- $\alpha$ and IFN-y) the two other members of chemokine family including CXCL1 and CXCL12 were also up-regulated [12].

It has also been evidenced that both CXCL1 and CXCL10 were significantly increased in several inflammatory and infectious disease states varying from T2D to multiple sclerosis. This is consistent with our findings regarding elevated CXCL1 and CXCL10 levels but not CXCL12 as a constitutive homoestatic CXC chemokine.

Therefore, according to the result of our study it can be concluded that AP and insulin by having anti-inflammatory properties may regulate $\mathrm{CXC1}$ and CXCL10 expression. Taken together, AP and insulin synergistically promote decreased symptoms of inflammation, probably via down regulation of these chemokines.

Our results demonstrated that the expression of CXCL12 remained unchanged during treatment with either insulin alone or combination of insulin and AP (Fig 3). The sustained level of CXCL12 could probably partially be explained by the fact that the gene structure of CXCL12 is a housekeeping form, CXCL12 is a TATA less and $\mathrm{GC}$ rich gene in which environmental factors are unable to effect its expression.

The consensus elements for the NF-KB, AP-1, and heat shock are lacking in the transcriptional regulatory site of CXCL12 gene which may also partially explain constitutive expression of its gene in presence of insulin and combination of $\mathrm{AP}$ and insulin. Inversely, in the regulatory region of both CXCL1 and CXCL10 the presence of consensus elements for intracellular signaling pathways, including MAPK, P38, and NF-KB and heat shock is validated by several researches. Thus, the regulatory effects of AP and insulin on these chemokine's regulation could probably be via affecting and activation of these intracellular pathways. One other reasonable fact by which could be speculated mechanism(s) involved in reduced chemokines, could be decreased levels of inflammatory molecules such as IL-1B, TNF- $\alpha$ and IFN-y following decreased inflammation symptoms [12]. Finally, based on the results of this investigation, we can conclude that probably $A P$, insulin and their combination indirectly control chemokines expression via lowering inflammation and also controlling its related symptoms. In another viewpoint, it is now well evidenced that specific receptors for CXCL1, CXCL10, and CXCL12 (CXCR1, CXCR3, and CXCR4, respectively), are present on endothelial cell [12]. Therefore, constitutive expression of CXCL12 and also massive expression of CXCL1 may probably be related to the specific role played by these chemokines in the processes of neovascularlization and formation of new capillary system via their angiogenesis properties regardless of their roles in inflammation [13]. However, CXCL10 exhibit angiostasis effects and inhibits formation of new vessels, thus, probably AP facilitates generation of the new capillary system through inhibition of CXCL10 expression [14]. Our findings also demonstrated that neutrophils are recruited more to the lesions of DM wounds and because CXCL1 is specific recruiter of neutrophils, it appears that CXCL1 is more functional and effective and recruited its respected cell type to the lesions. Moreover, the level of CXCL1 is also more than the other chemokines and increased neutrophil numbers may also at least partially be the result of the elevated expression of CXCL1 in comparison to the other chemokines determined in this study. It appeared that both leukocyte cell types (neutrophils and lymphocytes) migrated in response to wound-derived chemokines in stzinduced diabetic rats and mediated by CXC chemokines expression. Thus, leukocytes recruitment was decreased in conditions that chemokines (especially CXCL1 and CXCL10) expression was low (by immune blotting) (Figure 1,3). Therefore, it is likely that expression of studied chemokines in our investigation modulates leukocytes chemoattraction and probably in turn DM complications.

\section{CONCLUSION}

Overall, the results presented in this study show that $\mathrm{AP}$, due to its richness in some natural compounds such as coumarin and flavonoids, 
contributes to the process of chemokines expression. The drug probably affects two key etiological factors, namely, angiogenesis and inflammation, which are involved in pathogenesis of diabetes mellitus and its associated complications, at least partially, via balancing chemokines expression.

\section{ACKNOWLEDGEMENT}

The authors wish to appreciate Rafsanjan University of Medical Sciences, Iran for financial support.

\section{REFERENCES}

1. Arababadi MK, Nosratabadi R, Hassanshahi G, Yaghini $N$, Pooladvand $V$, Shamsizadeh A, Hakimi $H$, Derakhshan R. Nephropathic complication of type-2 diabetes is following pattern of autoimmune diseases? Diabetes Res Clin Pract 2010; 87(1): 3337.

2. Williamson JR, Kilo C. Capillary basement membranes in diabetes. Diabetes 1983; 32(Suppl 2): 96-100.

3. Lipsky BA, Berendt AR, Deery HG, Embil JM, Joseph WS, Karchmer AW, LeFrock JL, Lew DP, Mader JT, Norden $C$ et al. Diagnosis and treatment of diabetic foot infections. Clin Infect Dis 2004; 39(7): 885-910.

4. Eidi M, Eidi A, Zamanizadeh $H$. Effect of Salvia officinalis $L$. leaves on serum glucose and Insulin in healthy and streptozotocin-induced diabetic rats. J Ethnopharmacol 2005; 100(3): 310-313.

5. Farzamfar B, Abdollahi M, Ka, abinejadian S, Heshmat $R$, Shahhosseiny $\mathrm{MH}$. Sub-chronic toxicity study of a novel herbal-based formulation (Semelil) on dog. DARU 2008; 16(1): 15-19.

6. Hassanshahi G, Jafarzadeh A, Ghorashi Z, Zia Sheikholeslami N, Dickson AJ. Expression of IP-10 chemokine is regulated by pro-inflammatory cytokines in cultured hepatocytes. Iran J Allergy Asthma Immunol 2007; 6(3): 115-121.

7. Derakhshan R, Arababadi MK, Ahmadi Z, Karimabad $M N$, Salehabadi VA, Abedinzadeh $M$,
Khorramdelazad $H$, Balaei $P$, Kennedy $D$, Hassanshahi G. Increased circulating levels of SDF-1 (CXCL12) in type 2 diabetic patients are correlated to disease state but are unrelated to polymorphism of the SDF-1beta gene in the Iranian population. Inflammation 2012; 35(3): 900-904.

8. Newcomer CE. The evolution and adoption of standards used by AAALAC. J Am Assoc Lab Anim Sci. 2012; 51(3): 293-297.

9. Behzad-Behbahani A, Yaghobi R, Sabahi F, Rostaei MH, Alborzi A. Improvement in isolation of human peripheral blood leukocyte subpopulations: application in diagnosing human cytomegalovirus infection in bone marrow transplant patients. Exp Clin Transplant. 2005; 3(1): 316-319.

10. Hassanshahi G, Patel SS, Jafarzadeh AA, Dickson AJ.Expression of CXC chemokine IP-10/Mob-1 by primary hepatocytes following heat shock. Saudi Med J 2007; 28(4): 514-518.

11. Ghanim H, Korzeniewski K, Sia CL, Abuaysheh S, Lohano $T$, Chaudhuri A, Dandona P. Suppressive effect of Insulin infusion on chemokines and chemokine receptors. Diabetes care 2010; 33(5): 1103-1108.

12. Hassanshahi $G$, Jafarzadeh $A$, James Dickson $A$. Expression of stromal derived factor alpha (SDF-1 alpha) by primary hepatocytes following isolation and heat shock stimulation. Iran J Allergy Asthma Immunol 2008; 7(2): 61-68.

13. Hassanshahi G, Arababadi MK, Khoramdelazad $H$, Yaghini N, Zarandi ER. Assessment of CXCL12 (SDF-1alpha) polymorphisms and its serum level in posttransfusion occult HBV-infected patients in Southeastern Iran. Arch Med Res 2010; 41(5): 338342.

14. Azin H, Vazirinejad R, Ahmadabadi BN, Khorramdelazad $H$, Zarandi ER, Arababadi MK, Karimabad MN, Shamsizadeh A, Rafatpanah H, Hassanshahi G. The SDF-1 3'a genetic variation of the chemokine SDF1alpha (CXCL12) in parallel with its increased circulating levels is associated with susceptibility to MS: a study on Iranian multiple sclerosis patients. J Mol Neurosci 2012; 47(3): 431-436. 